\title{
Artificial Neural Network Model for Estimation of Suspended Sediment Load in Krishna River Basin, India
}

\author{
Vinay Kumar Rayaprol, Penke Satyannarayana, Arvind Yadav, Siva Ranjini, Uppara Geethika
}

\begin{abstract}
The correct assessment of amount of sediment during design, management and operation of water resources projects is very important. Efficiency of dam has been reduced due to sedimentation which is built for flood control, irrigation, power generation etc. There are traditional methods for the estimation of sediment are available but these cannot provide the accurate results because of involvement of very complex variables and processes. One of the best suitable artificial intelligence technique for modeling this phenomenon is artificial neural network (ANN). In the current study ANN techniques used for simulation monthly suspended sediment load at Vijayawada gauging station in Krishna river basin, Andhra Pradesh, India. Trial \& error method were used during the optimization of parameters that are involved in this model. Estimation of suspended sediment load (SSL) is done using water discharge and water level data as inputs. The water discharge, water level and sediment load is collected from January 1966 to December 2005. This approach is used for modelled the SSL. By considering the results, ANN has the satisfactory performance and more accurate results in the simulation of monthly SSL for the study location.
\end{abstract}

Keywords: Artificial neural network; Water discharge; Water level; Suspended sediment load; Krishna River.

\section{INTRODUCTION}

Rivers are the dynamic geological agent that contains the suspended sediment load and transfer the weathered materials into the ocean. The sediment's transport mechanism in a stream framework is a complex non-linear procedure which includes the interchange of the parameters of hydrological and geological changing in reality terms like space and time. Information on sediment's transportation alongside estimation and prediction of sediment's focus has many suggestions in the management of land use, water resources, regional risks, and harmfulness that will be caused to the structures of engineering coming about because of the

Revised Manuscript Received on January 05, 2020.

Dr. Penke Satyanarayana, Department of Electronics and Computer Engineering, Koneru Lakshmaiah Education Foundation, Vaddeswaram, AP, India. E-mail:satece@kluniversity.in

Vinay kumar rayaprol, Department of Electronics and Communication Engineering, Koneru Lakshmaiah Education Foundation, Vaddeswaram, AP, India. E-mail:160040765@kluniversity.in

Uppara geethika, Department of Electronics and Communication Engineering, Koneru Lakshmaiah Education Foundation, Vaddeswaram, AP, India. E-mail:160040257@kluniversity.in

Siva ranjani, Department of Electronics and Communication Engineering, Koneru Lakshmaiah Education Foundation, Vaddeswaram, AP, India. E-mail:160040765@kluniversity.in

Dr. Arvind Yadav, Department of Electronics and Computer Engineering,.Koneru Lakshmaiah Education Foundation, Vaddeswaram, AP, India. E-mail: arvindyadav@kluniversity.in morphological development of the stream bed. As there is no exact model produced for assessing this procedure has increased all inclusive acknowledgment and there is a developing requirement for advancement of minimal list experiential methodologies that are receptive to catchment qualities, climatic elements, and the antecedent conditions. The advancement of the sediment rating curve, which is used by a wide margin the most regular technique for estimation of the concentration of sediment, supposedly results in under-prediction and unsatisfactory results(Yadav et al 2017,2018). A few numerical revisions have been applied to the fundamental linear regression conditions to solve this issue and nonlinear regression conditions have additionally been advanced (Crowder et al., 2007; Holtschlag, 2001, Chaube 2017, Chaube 2018a,b). The multiple linear regression technique can not solve the complex non linear behavior of sediment. It can handle the linear type relationship. Artificial intelligence techniques were applied successfully by many researchers to solve complex non-linear worldwide problems in hydrology and other domains (Chakravarti and Jain 2014; Kebed et al. 2017; Chakravorti et al 2017a; Chakravorti and Das 2017b; Yadav et al. 2017, 2018; Husain and Jayanthi 2018; Patel et al. 2018; Patel et al. 2019a,b). Various researchers have been used artificial intelligence for sediment load estimation in river basin system (Kebed and Chakravarti 2017; Yadav et al 2018, Yadav 2019). Numerous investigations have shown the potential advancement of Artificial Neural Networks (ANNs) in demonstrating the sediment. The strategy of utilizing ANNs to model sediment's esteems has also been applied by various researchers. Further, expectations dependent on ANN models for processing the grouping of suspended sediment materials are found in the current study Previously, ANNs have commonly been applied in displaying geo-hydrological factors utilizing the continuous time arrangement information of long length durations . In the current study, an exertion has been made to see various changes in the SSC with Q utilizing ANNs for the Krishna River, Ganges Basin, basing on the information acquired from the Central Water Commission (CWC), Government of India. The problem has very high significance as still now no study on sediment's prediction for the Krishna River has been done.

\section{STUDY AREA}

Krishna river is selected as study area. Krishna River is a peninsular river. 
It is a south Indian state river. It flows in four states like Telangana, Karnataka, Maharsahtra and Andhra Pradesh. Earlier it flows in Karnataka. It is also known as Krishnaveni. It is the fourth biggest river in terms of water inflows and river basin area.

The flow of Krishna river started from Maharashtra,then it reaches to Telangana and then it covers Karnataka and finally it reaches Bay of Bengal. It originates from Mahabaleswar in Maharashtra. The mouth of Krishna river is in Andhra Pradesh and the place is known as Hamsaladeevi. Gataprabha river, Malaprabha river, Tungabhadra river, Bhima river and Musi river are the tributaries of Krishna river. On the right side it has venna, kogna, panchganga and Dudhaga and on the left side it has the Bhima,Peddavag,Musi,paleru and Dindi.

The Tungabhadra river is the largest tributary of Krishna river and the drainage basin is measuring $71,417 \mathrm{~km} 2$. Bhima is the longest tributary of Krishna river. Panchaganga,Warna and Yerla are the three tributaries meet Krishna river near sangli. These are the very holy places and 258,948 is the area that has been extended by the Krishna basin. Location of Vijayawada gauge station and Krishna river basin are given in Figure 1.

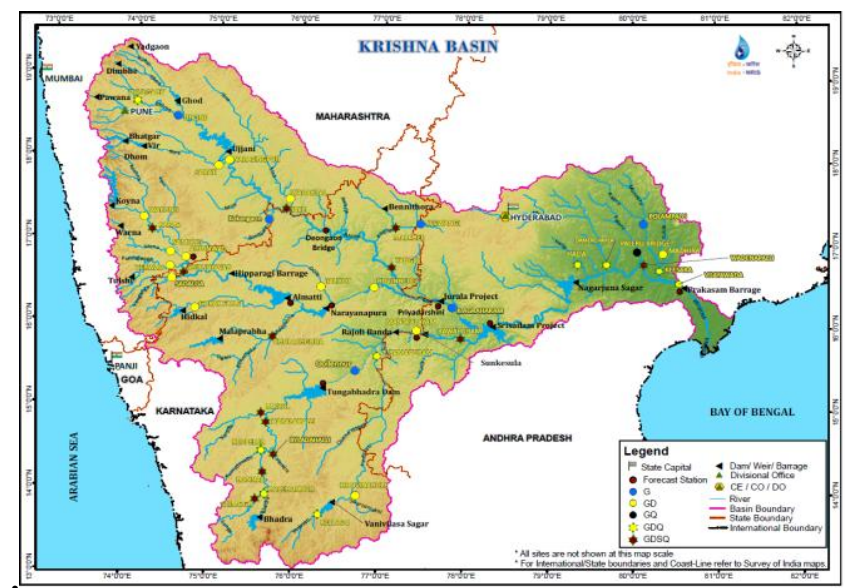

Figure 1. the above map represents the of the Krishna basin area indicating major streams

\section{METHODOLOGY}

\section{A. Artificial neural networks}

An ANN is dynamic system which is widely capable to incapturing linear and nonlinear interactions by proper learni -ng of patterns that are obtained from output and input (Haghizade et al. 2010) .Over past 20 years, mainly based models of artificial neural networks have seen the growing performance of hydrological system simulation(Singh and Panda 2011). Multiple layer perception (MLP) artificial neural network could be an artificial neural feed-forward nural network to the associated which are considered as input and hidden associated with the output layer. Every layer includes an accurate array of neurons performing related activation. According to the input and target parameters, the count of neurons present in the input layer and the count of neurons present in output layer should be equal. The error statistics will determine the number of neurons in the secret layer. Theoretical experiments indicated for approximation the number of hidden layers can be one for avoiding the complexity of the network. The under lying layer is utilized to stop network's complexes from growing(Cybenko 1989; Hornik et al. 1989). Every neural network will be trained in the hidden layer under many structures with totally different neuron numbers. In MLP, each layer connected with the total input into a degree of activation . that is determined by the activation. Figure 1 demonstrates the artificial neural network model for the sediment load's estimation.two different types of algorithms were used for the training of this model. such as feedforward back propagation (Pramanik , Panda 2009). Due to its fast response, the FFBP-LM algorithmic program is useful for the development of MLP neural network model. For a supervised algorithmic program, it is preferably a primary option, even if it needs additional memory. The weights allocated will be given for managing neural network later generate previous equivalent weights. The parameters have been modified to know the outcome of estimation of sediment yield. input parameters. The updated weight law of the algorithmic program development process from the steepest descent and the methodology of Newton (Hagan and Menhaj 1994) as follows:

$$
W_{k+1}=W_{k}-\left(J^{T} J+\mu I\right)^{-1} J^{T} e
$$

Where in the above equation $\mathrm{j}$ indicate the matrix of jacobian e indicates the error matrix. I indicates the matrix of identity. The parameter mu has the significant effect on the coefficient of combination and process of learning

\section{B. Data Processing}

Once neural systems get prepared, information we know the basic unit of data standardization and division area because information and yield information has various units and numerous range. The standardization of knowledge will be useful for the removal of variable's dimension and to preserve their individuality throughout the preparation of the models. This thoroughbred handling and assembly was fast $\mathrm{w}$ hen getting ready for the minimal forecastfailure (Rojas 199 6). Data standardization was achieved between 0 and 1 . The parameters which are considered as the inputs and the outputs in the current reach.standardization was performed.The knowledge was di videddata and used for training, validation and testing of mo dels to build generalized and reliable models for predicting $s$ ediment yield. Applied at the training of model testing and for making the validity. The data obtained from the training will be used to train the network of the neuron. while knowledge of validation is used to prevent ove rerfitting. Theknowledge of testing was regarded as hidden $\mathrm{i}$ nformation wherever the quality of the model is often checked.

\section{RESULTS AND DISCUSSION}

MATLAB software was used for the development of this model., and the data which is used for the ANN model development is the normalized. The neurons which are considered as inputs and the neurons which are considered as outputs and neurons of single underlying layer are the main objectives to develop the modeling of network of neurons 
with feedforward back propagation back propagation with Levenberg Marquardt (FFBP-LM) for estimation sediment's yield in Krishna river basin, Andhra Pradesh, India. Some methods were used to estimate the count of nodes under hidden layer are selected as 18. In the optimized model of FFBP-LM , tan sigmoidal function is used in the hidden layer and the pure linear activation function of linear activation in the layer that considered as output. It was recognized that the maximum value of $\mu$ is 0.006 . The variation in gradient, $\mu$ and validation check in training phase are given in figure 2.

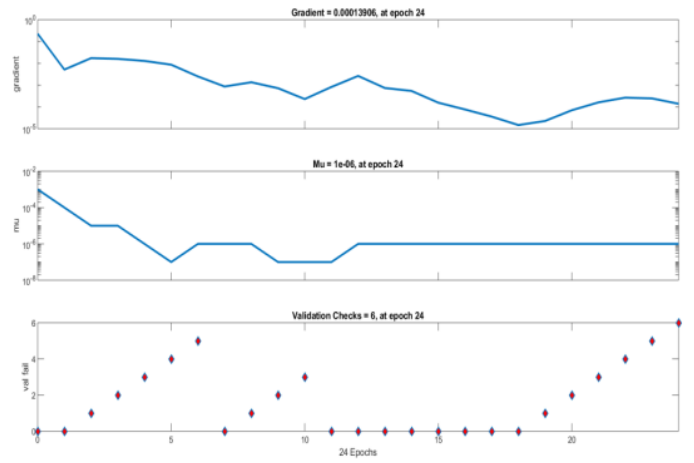

Figure 2. Variation of ANN model parameters in training phase

The 1:1 line is the bisector line (45 degree line), where we got the equal values for the observed and predicted numerical. If scatter points lies closely along this line then the values which are observed and predicted are appropriately equal, that indicates that model is perfect. Also, it is observed that the model is able to capture the low sediment's yield that was suspended as it is very clear in the plot which was scattered where negative value is not estimated during low suspended sediment data. the observations from FFBP-LM like sediment's yield that was suspended which are having low high and medium magnitudes was nearer to values that are observed (Figure 3). It is observed from the scatter plots (Figure 4 and Figure 5) that the values obtained from predictions and observations of the FFBP-LM were nearer to the inclination of 45 degrees (1:1), and values from the observations of sediment are closest to predicted values. The ANN estimated sediment load are very close to observed in the scatter plots(Figure 3). It is seen from both the plots that the values of sediment's yield that is suspended from estimation by the ANN model is closest to the observed data(Figure 3, Figure 4and Figure 5). Thus it is observed that the ANN model has capability to estimate at low, medium and high suspended sediment yield. It has more generalization capability. It is provided satisfactory performance for estimation of suspended sediment yield. It indicates that highly complex nonlinear sedimentations process has occurred but which is captured by the ANN model and generated positive suspended sediment yield values.

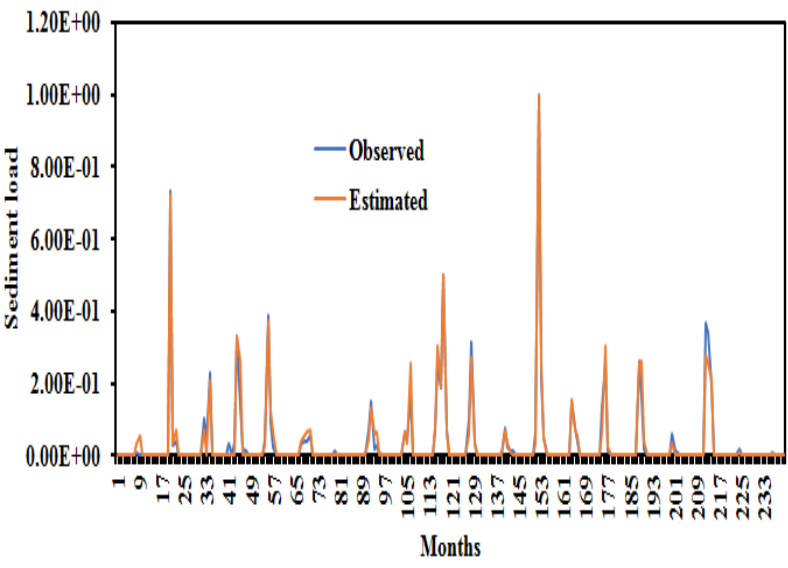

Figure 3. Difference among the values of observations and predictions sediment's yield of FFBP-LM model.
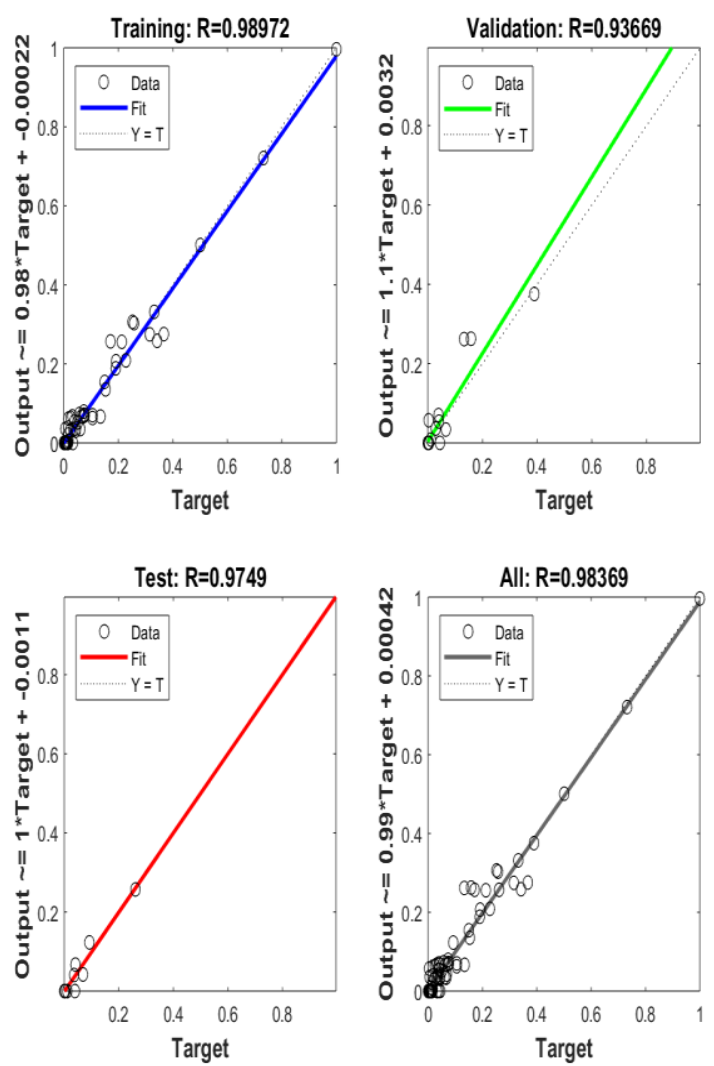

Figure 4. plots that are scattered of normalized values from the observations and predictions sediment's yield of a FFBP-LM model. 


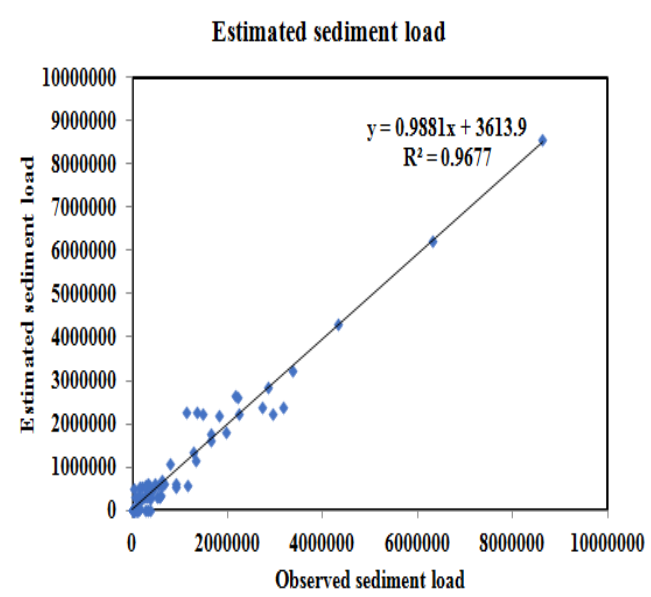

Figure 5. Scattered plots of values from the observations and predictions sediment's yield of a FFBP-LM ANN model.

\section{CONCLUSION}

The present study has exhibited the estimation and determining of suspended sediment yield in the Krishna river basin, India by utilizing the ANN model. The water discharge and water level data is utilized as information parameters in the models from Vijayawada gauge station. It was plainly seen that water release and water level are the most predominant controlling parameters of suspended sediment in the Krishna river. The exhibition and the speculation ability of ANN model is agreeable which may be because of thought of water release as information variable. The ANN model provided real estimation of suspended sediment yield, at lower observed sediment which produced positive suspended sediment. Since the sediment yield estimation can't be negative. The ANN model is provided the real positive estimate of size of low, medium and high. This sediment estimation will be valuable for better water for the plan of dams, channels, trenches, spans, water treatment forms, stream geomorphology, and valuation of water immaculateness issues in the Krishna river basin, India. It is very interesting to note that the predicting, the ANN, model always provides positive sediment value even if suspended sediment yield is zero or close to zero at Vijayawada gauging station in the Krishna basin. The information utilized is from one gage station and further procedures take more information from various gauge stations might be required to strength conclusions. In future research, various types of ANN will be analyzed and new datasets will be use before to the models.

\section{REFERENCES}

1. Chakravarti, A., Joshi, N., and Panjiar, H., 2015. Rainfall runoff modeling using artificial neural networks. Indian Journal of Science and Technology, Vol. 8(14), pp 1-7, ISSN - 0974-5645.

2. Chakravorti, Tatiana, and Pradipta Kishore Dash. "Multiclass power quality events classification using variational mode decomposition with fast reduced kernel extreme learning machine-based feature selection." IET Science, Measurement \& Technology 12.1 (2017a): 106-117.

3. Chakravorti, Tatiana, Rajesh Kumar Patnaik, and Pradipta Kishore Dash. "Detection and classification of islanding and power quality disturbances in microgrid using hybrid signal processing and data mining techniques." IET Signal Processing 12.1 (2017b): 82-94.
4. Chaube, A.K, Ishan, A., Ajay, J., Munise, M., Demirbas, D., Dey, D. 2018a. Dual - axis buckling of laminated composite skew hyperbolic paraboloids with openings, J. Brazilian Soc. Mech. Sci. Eng., 40, 490

5. Chaubey, A.K., Kumar, A., and Chakrabarti, A., 2018b.Novel shear deformation model for moderately thick and deep laminated composite conoidal shell, Mech Based Des Struc., vol. 46, no. 5, pp. 650-668.

6. Chaubey, A.K., Kumar, A., and Chakrabarti, A.,2017. Vibration of laminated composite shells with cutouts and concentrated mass, AIAA Journal, vol 56, no 4, pp.1662-1678.

7. Cybenko, G., 1989. Approximation by superpositions of a sigmoidal function. Math Control Signal 2:303-314

8. Dabbakuti, J.R.K. Jacob, A., Rao, V.V, ; Kumar, R.K. Implementation of IoT Analytics Ionospheric Forecasting System based on Machine Learning and ThingSpeak. IET Radar, Sonar \& Navigation, DOI: 10.1049/iet-rsn.2019.0394, 20 November 2019.

9. Hornik, K., Stinchcombe, M., White, H., 1989, Multilayer feed forward networks are universal approximators. Neural Netw 2:359-366.

10. Hussain, M.A., and Jayanthi, A. Novel Approach Certificate Revocation in MANETusing Fuzzy Logic. Indonesian Journal of Electrical Engineering and Computer Science, Vol.10, No.2 May 2018.

11. Kebede, M., Chakravarti, A. and Adugna, T., 2017. Stream flow and land use land cover changes in Finchaa Hydropower, Blue Nile river basin, Ethiopia" International Journal of Civil, Structural, Environmental, Infrastructures Engineering, Research \& Development (IJCSEIERD), 7, 1-12, ISSN:2249-7978.

12. Kebede, M., Chakravarti, A., 2017. Sediment yield assessment and mitigation measures in Finchaa Watershed, Ethiopia. International Journal of Engineering Research and Technology (IJERT), 6, Issue 08 , PP 220-226, ISSN: 2278-0181.

13. Mathematical Model in Mahanadi River Basin, India, Journal of Sustainable Water Resource Management, 4(4), 745-759.

14. Patel, A.K., Chatterjee, S. and Gorai, A.K., 2018. Development of an expert system for iron ore classification. Arab. J. Geosci, 11(15), p.401.

15. Patel, A.K., Chatterjee, S. and Gorai, A.K., 2019a. Development of a machine vision system using the support vector machine regression (SVR) algorithm for the online prediction of iron ore grades. Earth Sci. Inform, 12(2), pp.197-210.

16. Patel, A.K., Chatterjee, S. and Gorai, A.K., 2019b. Effect on the Performance of a Support Vector Machine Based Machine Vision System with Dry and Wet Ore Sample Images in Classification and Grade Prediction. Pattern Recogn and Image Anal, 29(2), pp.309-324.

17. Pramanik N, Panda RK (2009) Application of neural network and adaptive neuro-fuzzy inference systems for river flow prediction. Hydrol Sci J 54(2):247-260.

18. Tang Z, De Almeida C, Fishwick PA et. 1991. Time series forecasting using neural networks vs Box-Jenkins methodology. Simulation 57:303-310

19. Yadav, A., Chatterjee, S., Equeenuddin, S.M., 2017. Prediction of Suspended Sediment Yield by Artificial Neural Network and Traditional Mathematical Model in Mahanadi River Basin, India, Journal of Sustainable Water Resource Management, 4(4), 745-759.

20. Yadav, Arvind, Snehamoy Chatterjee, and Sk Md Equeenuddin. "Suspended sediment yield estimation using genetic algorithm-based artificial intelligence models: case study of Mahanadi River, India." Hydrological sciences journal 63.8 (2018): 1162-1182.

21. Yadav, Arvind. Estimation and Forecasting of Suspended Sediment Yield in Mahanadi River Basin: Application of Artificial Intelligence Algorithms. Diss. 2019.

\section{AUTHORS PROFILE}

Vinay Kumar Rayaprol: I am a student of KL University studying in final year of B-Tech. I have done my specification in Artificial Intelligence Technologies. I have worked on application of artificial intelligence in river basin system as my minor project.

Dr. Penke Satyannarayana: Professor, Electronics and Computer Science, Koneru Lakshmaiah University, Vaddeswaram, India.Ph.D JNTU. AP, India. 
Siva Ranjini: I am a student of KL University studying final year belongs to Department of ECE , B-Tech. I have done my specification in Artificial Intelligence Technologies. I have worked on application of artificial intelligence in river basin system as my project.

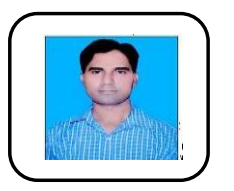

Dr. Arvind Yadav: I am Dr. Arvind Yadav, Assistant Professor, Electronics and Computer Engineering Department, KL university Vijayawada. I have completed Ph.D. from National Institute of Technology (NIT) Rourkela. mI like ton apply Artificial Intelligence Techniques in interdisciplinary fields. Recently, I have published the "Prediction of suspended sediment yield by artificial neural network and traditional mathematical model in Mahanadi river, India" paper in Springer. Recently, I have also published SCI free journal paper "Suspended Sediment Yield Estimation using Genetic Algorithm-based Artificial Intelligence Models in Mahanadi River “ of impact factor 2.3 in Taylor \& Francis. Many other papers which are based on application of artificial intelligence based techniques in interdisciplinary domains are under review in SCI journals. I have qualified GATE exam.

Uppara Geethika: I am a student of KL University studying final year belongs to Department of ECE , B-Tech. I have done my specification in Artificial Intelligence Technologies. I have worked on application of artificial intelligence in river basin system as my project. 